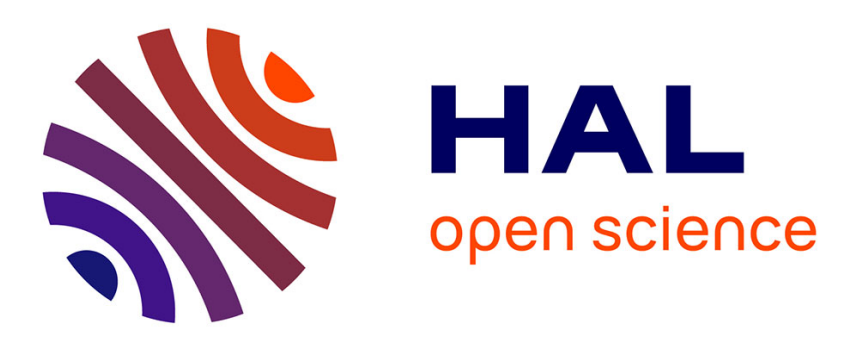

\title{
Emergence of spectral incoherent solitons through supercontinuum generation in photonic crystal fibers
}

\author{
B. Kibler, Christine Michel, Alexandre Kudlinski, B. Barviau, G. Millot,
} Antonio Picozzi

\section{- To cite this version:}

B. Kibler, Christine Michel, Alexandre Kudlinski, B. Barviau, G. Millot, et al.. Emergence of spectral incoherent solitons through supercontinuum generation in photonic crystal fibers. Physical Review E: Statistical, Nonlinear, and Soft Matter Physics, 2011, 84, pp.066605. 10.1103/PhysRevE.84.066605 . hal-00699911

\section{HAL Id: hal-00699911 \\ https://hal.science/hal-00699911}

Submitted on 22 May 2012

HAL is a multi-disciplinary open access archive for the deposit and dissemination of scientific research documents, whether they are published or not. The documents may come from teaching and research institutions in France or abroad, or from public or private research centers.
L'archive ouverte pluridisciplinaire HAL, est destinée au dépôt et à la diffusion de documents scientifiques de niveau recherche, publiés ou non, émanant des établissements d'enseignement et de recherche français ou étrangers, des laboratoires publics ou privés. 


\title{
Emergence of spectral incoherent solitons through supercontinuum generation in a photonic crystal fiber
}

\author{
B. Kibler,,${ }^{1,}$ C. Michel,${ }^{1}$ A. Kudlinski, ${ }^{2}$ B. Barviau,${ }^{1,2}$ G. Millot, ${ }^{1}$ and A. Picozzi ${ }^{1}$ \\ ${ }^{1}$ Laboratoire Interdisciplinaire Carnot de Bourgogne, UMR 5209 CNRS-Université de Bourgogne, FR-21078 Dijon, France \\ ${ }^{2}$ Université Lille 1, IRCICA (FR CNRS 3024), Laboratoire PhLAM (UMR CNRS 8523), FR-59655 Villeneuve d'Ascq, France
}

(Received 2 August 2011; published 19 December 2011)

\begin{abstract}
We report an experimental and numerical study of the spontaneous emergence of spectral incoherent solitons through supercontinuum generation in a two zero-dispersion wavelengths photonic crystal fiber. By using a simple experimental setup, we show that the highly nonlinear regime of supercontinuum generation is characterized by the emergence of a spectral incoherent soliton in the low-frequency edge of the supercontinuum spectrum. We show that a transition occurs from the discrete spectral incoherent soliton to its continuous counterpart as the power of the laser is increased. Contrary to conventional solitons, spectral incoherent solitons do not exhibit a confinement in the space-time domain, but solely in the frequency domain. These incoherent structures owe their existence to the noninstantaneous nature of the nonlinear Raman effect and, more specifically, to the causality property underlying the Raman response function.
\end{abstract}

DOI: 10.1103/PhysRevE.84.066605

PACS number(s): 05.45.Yv, 42.65.-k, 42.81.Dp

\section{INTRODUCTION}

During the last decade, the high nonlinearity of photonic crystal fibers (PCF) and the ability to readily engineer their dispersion characteristics have enabled the study of various new nonlinear effects $[1,2]$. In particular, the process of supercontinuum (SC) generation in a PCF has been extensively studied and distinct regimes have been identified depending on whether the PCF is pumped in the normal or anomalous dispersion regimes, or with short (subpicosecond) or long [picosecond, nanosecond, and quasi-continuous wave (cw)] pump pulses (see Ref. [3] for more details). The spectral broadening inherent to SC generation is usually interpreted through the analysis of the following main contributions: the four-wave mixing effect, the soliton fission, the Raman self-frequency shift, and the generation of dispersive waves [3-6].

In the case where the PCF is pumped with long pulses in the anomalous dispersion regime, the general physical picture is that modulation instability (MI) leads to the generation of a train of solitonlike pulses, which are known to emit (Cherenkov) radiation in the form of spectrally shifted dispersive waves. Moreover, as a result of the Raman effect, the generated optical solitons exhibit a self-frequency shift towards longer wavelengths. The same picture holds when the PCF exhibits two zero-dispersion wavelengths (ZDWs): The Raman frequency shift of the solitons is eventually arrested in the vicinity of the second $\mathrm{ZDW}$, so that the SC spectrum is essentially bounded by the corresponding dispersive waves [5,6]. Accordingly, in all these regimes the existence of coherent soliton structures plays a fundamental role in the process of SC generation.

This usual physical picture of SC generation changes profoundly when the pump power of the long pulses injected in the PCF is increased in a substantial way. In this case, the spectral broadening process is essentially dominated by the

\footnotetext{
*bertrand.kibler@u-bourgogne.fr
}

combined effects of the Kerr nonlinearity and higher-order dispersion, i.e., by four-wave mixing processes [7]. More precisely, in this regime the rapid and random temporal fluctuations of the optical field prevent the formation of robust coherent soliton structures. Note that this is a rather general property of nonlinear wave systems: As the incoherence of the wave increases, the system becomes "too hot" to generate a coherent soliton structure (see, e.g., [8,9]). In this SC regime, the optical field thus exhibits an incoherent turbulent dynamics, in which coherent soliton structures no longer play any significant role. In the following we shall call this regime the "incoherent regime of SC generation" [10].

We have recently formulated a nonequilibrium kinetic description of this incoherent regime of SC generation on the basis of the weak-turbulence theory [10-12]. We showed that, in the conservative limit, i.e., neglecting the dissipative Raman effect, the four-wave mixing underlying the spectral broadening of the incoherent optical field can be described as a nonequilibrium thermodynamic process. In particular, when the PCF is characterized by two closely spaced ZDWs, SC generation can be interpreted as a consequence of the thermalization of the optical field [10-12], i.e., the spectral broadening results from the natural tendency of the optical field to reach the thermal equilibrium state that realizes the maximum of entropy [13-16]. More precisely, we showed that the generated SC spectrum can be characterized by an unexpected double-peak structure, which is nothing but the fundamental Rayleigh-Jeans thermodynamic equilibrium spectrum of light $[11,12]$.

This process of SC thermalization takes place in the conservative limit of the propagation of the optical field, so that its description does not account for the dissipative Raman effect. Actually, the analysis revealed that, besides the thermalized SC spectrum, the Raman effect is responsible for the generation of an incoherent structure in the low-frequency edge of the spectrum [11]. The numerical simulations showed that this incoherent structure exhibits qualitative properties very similar to those of spectral incoherent solitons (SIS). The SIS is a solitary wave structure that has been recently 
identified in Ref. [17]. It is of a fundamentally different nature than conventional optical solitons, which are inherently coherent localized structures. SISs are characterized by two important properties: (i) The optical field associated with a SIS is incoherent; (ii) contrary to conventional solitons, SISs do not exhibit a confinement of the optical field in the spatiotemporal domain, but exclusively in the spectral domain (also see [18]). More precisely, the partially coherent optical field associated with SIS is characterized by temporal fluctuations that are statistically stationary in time, so that the soliton behavior only manifests in the spectral domain, but not in the temporal domain. We underline that SIS can be sustained in both the normal and anomalous dispersion regimes. It is also important to recall that SISs find their origin in the property of causality that characterizes the Raman response function of the nonlinear material [17].

In a recent theoretical work we showed that SISs identified in Ref. [17] can also exhibit a discrete behavior [19]. In substance, discrete SISs are characterized by three incoherent spectral bands, whose frequencies refer to the central frequency $\left(v_{j}\right)$ and the corresponding Stokes $\left(v_{S}=v_{j}-v_{R}\right)$ and anti-Stokes $\left(v_{\mathrm{A}}=v_{\mathrm{j}}+v_{\mathrm{R}}\right)$ frequencies, where $v_{\mathrm{R}}$ is the Raman resonant frequency $\left(v_{\mathrm{R}} \approx 13.2 \mathrm{THz}\right.$ in silica fibers). As a result of the Raman effect, a new Stokes component is generated in the front of the soliton, which becomes in turn the central band, and finally the anti-Stokes band in the trailing edge of the soliton. In other terms, a new Raman band grows up by absorbing the previously generated spectral band, thus leading to a "discrete propagation" of the SIS toward the low-frequency components: The discrete SIS propagates without distortion in frequency space with a constant velocity, without emitting any apparent radiation [19]. In order to avoid confusion, in the following we shall term continuous SISs the spectral solitons identified in [17], so as to distinguish them from the discrete SISs recently predicted theoretically in [19]. The theory revealed that these kind of incoherent solitons can be described in detail by a rather simple kinetic equation (KE), which describes the evolution of the averaged spectrum of the incoherent optical field $[17,19]$. Note that the structure of this equation is analogous to a kinetic equation derived in plasma physics to describe weak Langmuir turbulence and stimulated Compton scattering [20,21].

Our aim in this article is to report the experimental observation of the emergence of both discrete and continuous SISs in the highly nonlinear regime of SC generation. An experimental procedure based on a cutback of the PCF with different values of the pump power has allowed us to perform detailed measurements of the evolution of the spectrum of the field during its propagation. The experimental results are in good agreement with the numerical simulations and thus provide a signature of the emergence of discrete and continuous SISs. In particular, in order to get physical insight into the generation of SISs, we performed simulations with different models characterized by different levels of approximations: (i) the generalized nonlinear Schrödinger equation (GNLSE), (ii) a reduced delayed nonlinear Schrödinger equation (RNLSE), and (iii) the weak-turbulence KE, which is the simplest model describing SISs [17]. The analysis reveals a remarkable agreement between the experimental results and the simulations of the KE, which thus gives experimental evidence of the emergence of SISs from the SC spectrum. Although discrete SISs are asymptotically generated, we show that in the first stage of propagation a transition occurs from discrete SISs to continuous SISs as the power injected into the PCF is increased, a remarkable feature that is described in detail by the KE.

Besides the context of SC generation, this work is also important from a broader perspective. The study of incoherent nonlinear optics is indeed attracting a growing interest in various different fields of investigation, including, e.g., wave propagation in homogeneous [22-29] or periodic media [30], nonlinear imaging [31], cavity systems [32-40], or nonlinear interferometry [41]. In particular, besides SC generation [10-12], the long-term evolution of partially coherent optical waves has been studied in various circumstances [23-29,42-47] and in various optical media characterized by different nonlinearities [24,28,46,47]. In particular, the long-term behavior of the field may be characterized by a process of thermalization. As mentioned above, thermalization of light manifests itself by means of an irreversible evolution of the optical field toward the thermodynamic equilibrium state, i.e., the Rayleigh-Jeans spectrum [13-16]. This process of relaxation to equilibrium may be interpreted intuitively as a consequence of the natural tendency of a closed system to increase its entropy, i.e., the " "amount of disorder" in the optical field. SISs are important from this perspective: In contrast with the natural process of thermalization, the optical field can self-organize into SISs, which thus constitute genuine nonstationary and nonequilibrium stable states of the incoherent optical field.

\section{EXPERIMENTAL CONFIGURATION AND NUMERICAL MODEL}

The configuration of the experiment is the same as that reported in [11]. Below we thus briefly sketch the experimental setup and the experimental procedure, and we refer the reader to Ref. [11] for more technical details. The main difference with respect to the experiment reported in [11] is related to the dispersion properties of the PCF. Contrary to [11] in which the analysis was focused on the properties of the SC spectrum, we are interested here in the dynamics of SISs generated in the low-frequency range of the SC spectrum. We have thus designed a PCF with a dispersion curve similar to that used in [11], except that the two ZDWs have been shifted towards lower wavelengths. In this way the emergence of SISs takes place closer to the laser pump frequency, which thus enables the characterization of SISs with a standard optical spectrum analyzer.

The pump laser frequency $\nu_{0}=282 \mathrm{THz}\left(\lambda_{0}=1064 \mathrm{~nm}\right)$ lies in the anomalous dispersion regime of the PCF, i.e., it is located between the two ZDWs. It is important to note that the two ZDWs are closely spaced in relation to each other, a feature that accelerates the development of the highly nonlinear and incoherent regime of SC generation [10-12]. Such closely spaced ZDWs can be obtained by using a microstructured cladding with a low pitch value. They are known to substantially increase the gain of the modulation instability (MI) thanks to the low value of the second-order dispersion coefficient and the high nonlinearity. The fiber that we used is a $21-\mathrm{m}-$ long PCF manufactured by IRCICA. The scanning 

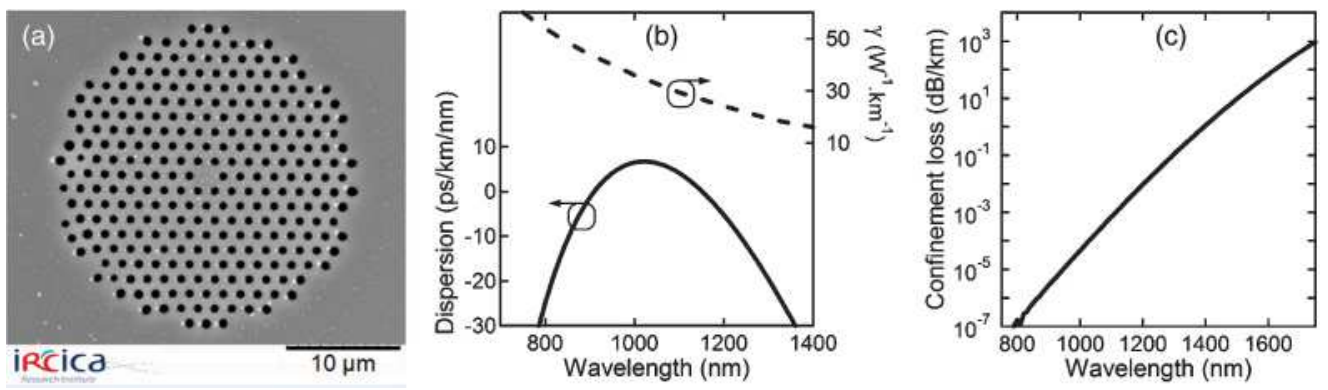

FIG. 1. (Color online) (a) Scanning electron microscope image of the PCF cross section. Calculated characteristics of the fundamental mode: (b) Dispersion curve (left axis) showing two zero-dispersion wavelengths located at 910 and $1152 \mathrm{~nm}$ and nonlinear coefficient (right axis), (c) confinement loss. The laser wavelength $\left(\lambda_{\mathrm{p}}=1064 \mathrm{~nm}\right)$ lies between the two dispersion wavelengths in the anomalous dispersion regime.

electron microscope image of the fiber cross section is illustrated in Fig. 1(a). The PCF has a core diameter of $\sim 2.3 \mu \mathrm{m}$, pitch $\Lambda=1.45 \mu \mathrm{m}$, and relative air hole diameter $d / \Lambda \approx$ 0.43 , which ensures the endlessly single-mode guidance. Linear and nonlinear properties related to the fundamental mode such as the dispersion profile, nonlinear coefficient, and confinement losses, were calculated using a commercial fully vectorial mode solver [see Figs. 1(b) and 1(c)]. The two ZDWs have been estimated to be $910 \mathrm{~nm}\left(v_{\mathrm{ZDW} 1}-v_{0} \approx 48 \mathrm{THz}\right)$ and $1152 \mathrm{~nm}\left(v_{\mathrm{ZDW} 2}-v_{0} \approx-22 \mathrm{THz}\right)$. At the pump wavelength, our PCF exhibits a low anomalous dispersion value of $-3.5 \mathrm{ps}^{2} / \mathrm{km}$ and its nonlinear parameter was calculated to be $\gamma=30 \mathrm{~W}^{-1} \mathrm{~km}^{-1}$. Fiber losses were also measured to be $\sim 0.04 \mathrm{~dB} / \mathrm{m}$ around the pump wavelength, except at
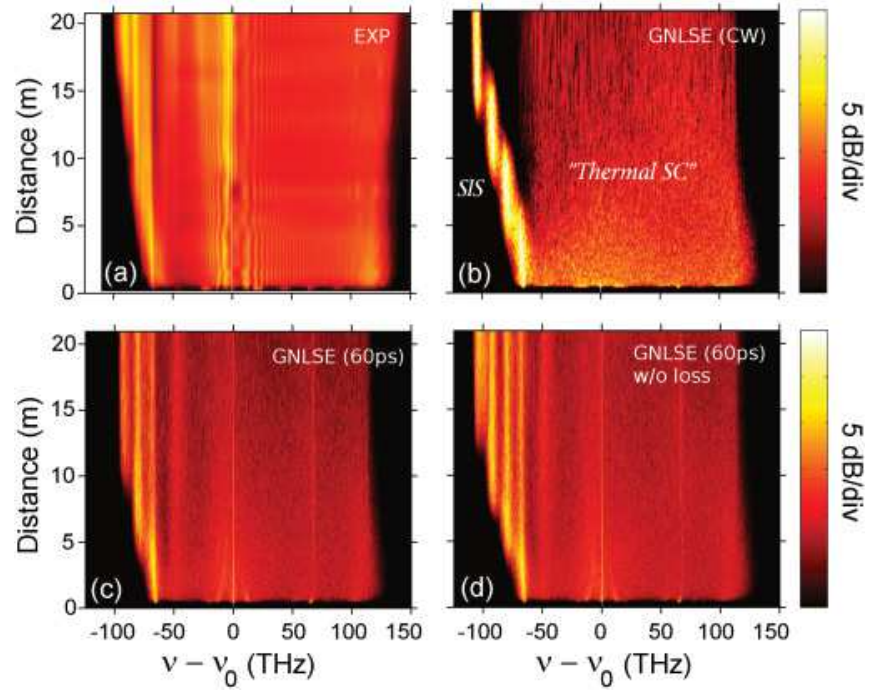

FIG. 2. (Color online) (a) Experimental results using a logarithmic intensity scale $(\mathrm{dB})$ to illustrate the spectral evolution as a function of propagation distance in our 21-m-long PCF, for an input peak power about $1.32 \mathrm{~kW}$. (b) Corresponding numerical simulations of the GNLSE (1) with the fiber characteristics of Fig. 1(b) and an input continuous wave. (c) Same as in (b) except that the initial condition is a Gaussian pulse of $60 \mathrm{ps}$, i.e., approximately ten times shorter than the experimental pulses. (d) Same as in (c), except that fiber losses are neglected $[\hat{\alpha}=0$ in the GNLSE (1)]. In (b) a discrete SIS moves away from the central part of the SC spectrum, which slowly relaxes toward thermal equilibrium [10-12].
$1380 \mathrm{~nm}$ due to the $\mathrm{OH}$ absorption peak [48] with losses up to $0.24 \mathrm{~dB} / \mathrm{m}$, while beyond $1600 \mathrm{~nm}$ confinement losses are dominant with values up to $1 \mathrm{~dB} / \mathrm{m}$. We made use of a low-cost commercial high-power subnanosecond pump delivering 660-ps pulses at $1064 \mathrm{~nm}$ with a peak power up to $14 \mathrm{~kW}$. This high-power quasi-cw pump allows us to investigate the highly nonlinear and incoherent regime of SC generation with pump powers exceeding $1 \mathrm{~kW}$.

We performed numerical simulations of the following generalized GNLSE, which is known to provide an accurate description of the propagation of a broadband optical field in a PCF [3]:

$$
\begin{aligned}
\frac{\partial A}{\partial z}= & i \sum_{m \geqslant 2} \frac{i^{m} \beta_{m}}{m !} \frac{\partial^{m} A}{\partial t^{m}}+i \gamma\left[1+i \tau_{s} \frac{\partial}{\partial t}\right] \\
& \times\left[A(z, t) \int_{-\infty}^{+\infty} R\left(t^{\prime}\right)\left|A\left(z, t-t^{\prime}\right)\right|^{2} d t^{\prime}\right]-\hat{\alpha} A,
\end{aligned}
$$

where $\gamma$ is the nonlinear coefficient at the pump frequency and $R(t)=\left(1-f_{\mathrm{R}}\right) \delta(t)+f_{\mathrm{R}} h_{\mathrm{R}}(t)$ is the usual nonlinear response function of silica fibers, which accounts for the instantaneous Kerr effect and the noninstantaneous Raman response function $h_{\mathrm{R}}(t)$. The GNLSE (1) describes self-steepening effects through the optical shock term (the term proportional to $\left.i \tau_{s} \partial / \partial \mathrm{t}\right)$, which accounts for the dispersion of the nonlinearity, i.e., the frequency dependence of the nonlinear coefficient. As usual in SC simulations, it is important to include higher-order dispersion effects in the model of Eq. (1) so as to provide a detailed description of the dispersion curve of the PCF. Finally, the last term in the GNLSE takes into account the losses of the PCF. The symbol $\hat{\alpha} A$ means that its Fourier transform is $\alpha(\omega)$ $A(z, \omega), \alpha(\omega)$ being the losses of the optical field vs frequency. For a complete discussion of the different terms of Eq. (1), we refer the reader to Ref. [3]. Note that, in order to compare the (single shot) numerical results with the spectra recorded experimentally, the numerical spectra have been smoothed by using the method described in Ref. [11]. We have verified that the evolution of the spectrum does not depend significantly on the noise superimposed on the input pulse.

\section{EXPERIMENTAL RESULTS AND COMPARISON TO SIMULATIONS}

In order to provide experimental evidence of the emergence of SISs through SC generation, it is important to record the 

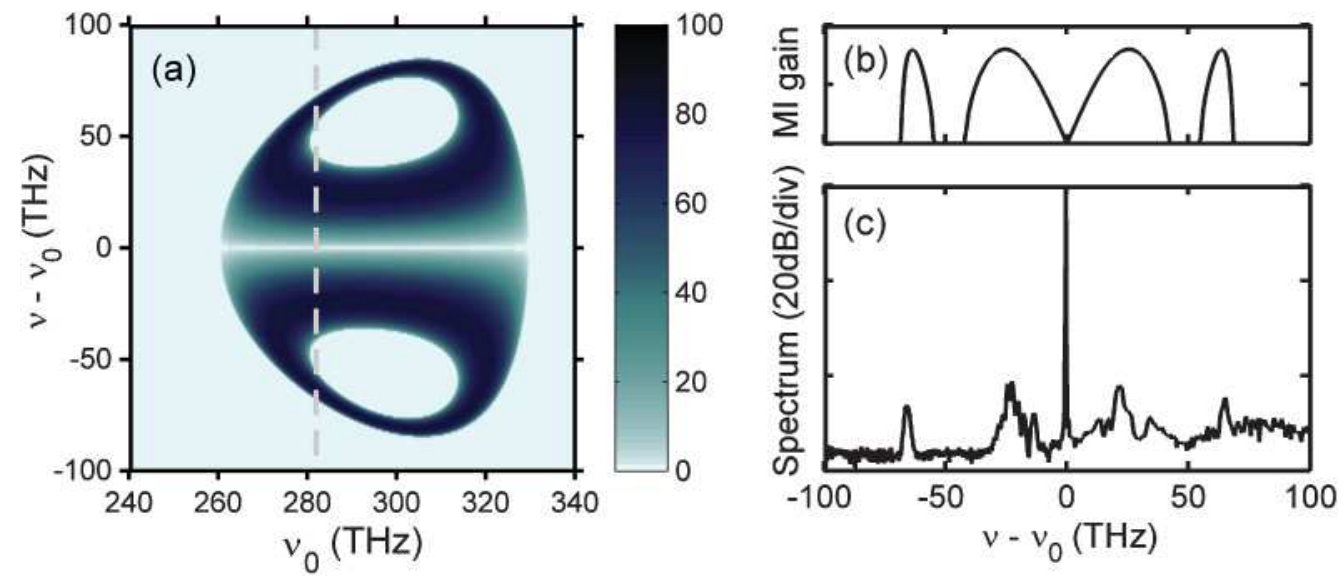

FIG. 3. (Color online) (a) Calculated MI gain $\left(\mathrm{m}^{-1}\right)$ bands as a function of pump wavelength for the dispersion curve of Fig. 1(b) and for an input peak power of $1.32 \mathrm{~kW}$. (The gray dashed line indicates our laser frequency). (b) MI gain calculated for our pump frequency from (a) showing two sets of bands, the first one at $\pm 22 \mathrm{THz}$, while the second one is located around $\pm 65 \mathrm{THz}$. (c) Experimental spectrum recorded after $20 \mathrm{~cm}$ of propagation in our PCF for an input peak power of $1.32 \mathrm{~kW}$.

spectrum of the field at different propagation lengths, while keeping fixed all other experimental parameters (e.g., injected power). For this purpose, we performed an experiment by cutting back the PCF length in increments of a few tens of centimeters. For each length we recorded the spectrum for three different values of the laser peak power, namely 1.32 , 2.22, and $4.52 \mathrm{~kW}$. We report in Fig. 2(a) the evolution of the spectrum along the $21 \mathrm{~m}$ of the PCF for a peak power of $1.32 \mathrm{~kW}$. We remark that each recorded spectrum does not correspond to a single shot spectrum, but to the average of a multitude of spectra (due to the long integration time of the spectrometer with respect to the pump repetition rate), which thus leads to a "partial smoothing" of the SC spectra (we refer the reader to [11] for details). We observe in Fig. 2(a) a rapid initial spectral broadening in the first meter of propagation in the PCF, which is due to the development of two sets of MI gain bands [also see Fig. 2(b)]. The fact that closely spaced ZDWs lead to the formation of a double set of MI gain bands has been recently studied theoretically in Ref. [49]. Figure 3(a) shows the scalar MI gain bands as a function of the pump frequency calculated from the dispersion properties of Fig. 1(b) using a $1.32 \mathrm{~kW}$ input power. We clearly note a qualitative agreement between the two sets of predicted MI gain bands at our pump frequency $(\sim 282 \mathrm{THz})$ and the experimental spectrum recorded after $20 \mathrm{~cm}$ of propagation [see Figs. 3(b) and 3(c)]. The first one generates frequencies around the pump frequency (at $22 \mathrm{THz}$ ), while the second one takes place around $65 \mathrm{THz}$ beyond the pump frequency in the normal dispersion regime. We note in Fig. 2(a) that the evolution of the spectrum exhibits a global asymmetric shape with respect to the carrier frequency of the laser, $v_{0}$. As discussed in our previous work [11], such asymmetry is in part due to the Raman effect, which leads to a permanent transfer of power toward the low-frequency components of the field. In particular, we showed that the Raman effect is responsible for the generation of an incoherent structure in the low-frequency edge of the SC spectrum which is reminiscent of SISs $[10,11]$. We may note in Fig. 2 that the MI band located at $-65 \mathrm{THz}$ plays a role analogous to a "seed" for the generation of the SIS.
This important observation will be the subject of a detailed discussion in Sec. IV.

To further analyze the evolution of the experimental spectrum, we report in Figs. 2(b)-2(d) a series of numerical simulations obtained by integrating numerically the GNLSE (1). These numerical simulations take into account the linear and nonlinear properties of the PCF discussed above through Fig. 1. In Fig. 2(b), the initial condition is a continuous wave, whose power corresponds to the peak power of the pulses used in the experiment $(1.32 \mathrm{~kW})$. A small amplitude noise has been superimposed to the continuous wave so as to initiate the MI process. The numerical simulation reveals the development of a highly incoherent regime of interaction, where rapid temporal fluctuations of the field prevent the formation of robust coherent soliton structures. As in our previous works $[10,12]$, we analyzed with care the evolution of the field $A(z, t)$ in many different cases and no persistent coherent soliton structures were identified. We remark in Fig. 2(b) that the spectrum of the field essentially splits into two components, i.e., a broad central part and a low-frequency branch that moves away from the central part of the spectrum. This low-frequency branch tends to evolve to a discrete SIS, which propagates in the spectral domain toward the low-frequency components. The discrete motion of the discrete SIS in frequency space is clearly visible. In particular, the frequency distance between adjacent discrete bands corresponds to the Raman resonant frequency ( $\nu_{\mathrm{R}} \approx 13.2 \mathrm{THz}$ ), a feature that we have also verified in the experimental spectrum [Fig. 2(a)]. However, one may notice in Fig. 2(b) that the propagation of the discrete SIS is not truly invariant, since its spectral bands become thinner and stretched during the propagation. This feature is exclusively due to the losses of the PCF. Indeed, numerical simulations identical to that reported in Fig. 2(b) have been realized in the lossless limit $[\hat{\alpha}=0$ in Eq. (1)]. They reveal the formation of a genuine discrete SIS that moves away from the SC spectrum and that propagates without distortion in frequency space over a broad spectral range that exceeds $10 v_{R}$, a remarkable feature illustrated in Fig. 1 of Ref. [19]. 
The comparison of Figs. 2(a) and 2(b) clearly reveals a substantial difference between the experimental results and the numerical simulation of the GNLSE (1). In the simulation the discrete SIS moves away from the central part of the SC spectrum, while in the experiment such a separation is not apparent. This aspect was studied in detail in our previous work [11]: The absence of a clear separation between the SIS and the SC spectrum is due to the envelope profile of the pulses delivered by our laser source. Indeed, we report in Fig. 2(c) a numerical simulation realized in the same conditions as in Fig. 2(b), except that the initial condition is not a continuous wave, but a 60-ps Gaussian pulse with the same peak power as in the experiment, $1.32 \mathrm{~kW}$. Note that this value of the pulse width is one order of magnitude smaller than that used in the experiment (660 ps). Because of the huge numerical time required by these simulations, we have not been able to perform simulations with the experimental values of the pulse widths. Nevertheless, the comparison of Figs. 2(b) and 2(c) clearly shows the impact of the limited pulse duration of the envelope profile. In particular, one may notice in Fig. 2(c) that the envelope profile induces a stretching of the spectral bands of the discrete SIS. This may be easily explained by the fact that the spectral shift induced by the Raman effect depends on the local value of the power on the pulse envelope: The Raman spectral shift on the peak of the pulse occurs before the corresponding spectral shift in the tails of the pulse, which merely explains the stretching of the spectral bands of the discrete SIS [Fig. 2(c)]. We corroborated this interpretation by performing a numerical spectrogram during the whole propagation of the field, whose analysis provided results similar to those obtained in Ref. [11] (see Fig. 6 in [11]).

We finally note that, although the separation between the discrete SIS and the central part of the SC spectrum is not apparent in the experiment, we can identify in Figs. 2(a) and 2(c) the development of a small trough of the spectrum at $-65 \mathrm{THz}$ from the laser frequency (i.e., $1380 \mathrm{~nm}$ ). One may ascribe such a spectral depletion to the fiber losses associated with the $\mathrm{OH}$ absorption peak [48]. However, we report in Fig. 2(d) the same numerical simulation realized in Fig. 2(c), except that we removed the fiber losses $[\hat{\alpha}=0$ in Eq. (1)]. It remarkably reveals that the evolution of the spectrum exhibits the same spectral trough at $-65 \mathrm{THz}$ from the laser frequency. This frequency precisely corresponds to the low-frequency edge of the central part of the SC spectrum [Fig. 2(b)], below which the emergence of the discrete SIS is clearly visible. The spectral trough identified experimentally at $-65 \mathrm{THz}$ can thus be attributed to the tendency of the spectral soliton to move away from the SC spectrum. In Sec. IV we corroborate this interpretation through the analysis of simple models of wave propagation.

\section{DISCRETE AND CONTINUOUS SIS}

Before entering into the details of the experimental results, we would like to briefly comment on the general conditions required to generate a SIS. In substance, an initial incoherent optical field characterized by a (quasi-) stationary statistics and a spectral width not negligible with respect to the Raman spectral bandwidth should be transformed into a SIS during the evolution, irrespective of the sign of the dispersion regime that can be either normal or anomalous. It is also important to verify that the incoherent optical wave evolves in the weakly nonlinear regime [13-16], i.e., its time correlation $t_{c}$ has to be short enough to make linear dispersive effects dominant with respect to nonlinear effects, $L_{D}=t_{c}^{2} / \beta_{2} \ll L_{\mathrm{NL}}$, where $L_{\mathrm{D}}$ and $L_{\mathrm{NL}}$ are the dispersive and nonlinear characteristic lengths, respectively (see, e.g., [48]). The assumption of a weakly nonlinear regime plays an essential role in the derivation of the KE (4), a feature that will be discussed in Sec. IV B. In particular, the KE reveals that SISs can be sustained in both the normal and anomalous dispersion regimes.

We analyze in this section the influence of the power of the laser source on the dynamics of the emergence of SIS from the $\mathrm{SC}$ spectrum. We show that in the first stage of propagation, a transition occurs from the discrete SIS toward the continuous SIS as the power injected in the PCF is increased. We report in Figs. 4(a)-4(c) the experimental spectrum recorded after $75 \mathrm{~cm}$ of propagation through the PCF for three different values of the input peak power. We first note that $75 \mathrm{~cm}$ of propagation are sufficient for a complete development of the MI bands. As anticipated in the previous section, the lowest-frequency MI band refers to the low-frequency edge of the SC spectrum and thus plays the role of a seed for the generation of SISs. It is important to note that, as in the standard MI process [48], the spectral bandwidth of the MI gain increases as the pump power is increased (in particular, it can lead to an overlapping of the two sets of MI gain bands), a feature which is also clearly visible in Fig. 4. Accordingly, the spectral seed of the SIS becomes larger as the injected power increases. As we shall see below, this spectral broadening is responsible for the transition from the discrete to the continuous SIS.
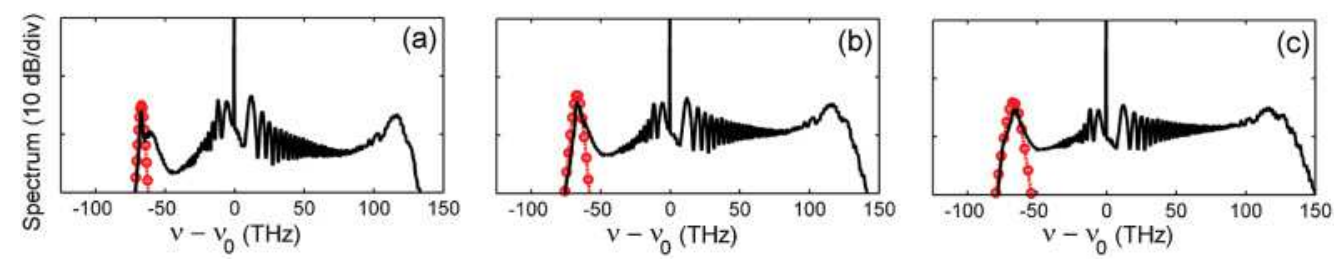

FIG. 4. (Color online) (Black solid line) Experimental spectra recorded after $75 \mathrm{~cm}$ of propagation for three distinct values of input peak power: (a) $1.32 \mathrm{~kW}$, (b) $2.22 \mathrm{~kW}$, and (c) $4.52 \mathrm{~kW}$. The red dashed lines with circles are the secant-hyperbolic fits of the low-frequency edge of the SC spectra, which play the role of seeds for the generation of SISs. The spectral widths and powers of the corresponding adjusted functions are, respectively, (a) $3 \mathrm{THz}, 150 \mathrm{~W}$, (b) $6 \mathrm{THz}, 250 \mathrm{~W}$, and (c) $9 \mathrm{THz}, 350 \mathrm{~W}$. Note that the spectral modulations on the central part of the spectra stem from the recording system used in our experiment, and refer to experimental artifacts [see also Figs. 5(a), 6(a), and 7(a)]. 


\section{A. Reduced delayed NLSE model}

To analyze this transition in detail, we fitted the lowfrequency MI band recorded experimentally by a secanthyperbolic function for each of the three input powers. The corresponding fits are shown in red in Fig. 4 and their characteristics (spectral width and peak power) are given in the figure caption. We considered these sech-fitting functions as the initial condition of the optical wave and we performed numerical simulations with a reduced model equation with delayed nonlinear response, i.e., the Raman response. This reduced model only retains the essential ingredients responsible for the existence of SIS, namely the lowest-order dispersion effect and stimulated Raman scattering [17,19]:

$$
\begin{aligned}
\frac{\partial A}{\partial z}= & -i \frac{\beta_{2}}{2} \frac{\partial^{2} A}{\partial t^{2}} \\
& +i \gamma\left[A(z, t) \int_{-\infty}^{+\infty} f_{\mathrm{R}} h_{\mathrm{R}}\left(t^{\prime}\right)\left|A\left(z, t-t^{\prime}\right)\right|^{2} d t^{\prime}\right] .
\end{aligned}
$$

We emphasize that in this equation we only retained the delayed nonlinear response, i.e., we neglected the instantaneous Kerr contribution. Equation (2) will be termed RNLSE so as to distinguish it from the GNLSE (1). The RNLSE (2) was shown to describe the essential properties of both discrete and continuous SISs. We refer the reader to Ref. [19] for a complete discussion of SISs in the framework of the RNLSE (2). To be consistent, an average value of $\beta_{2}$ $\left(160 \mathrm{ps}^{2} / \mathrm{km}\right)$ was considered in the RNLSE (2). It represents a good approximation of the dispersion value of the PCF in the frequency range swept by the SIS in the experiment. We made use of the following analytical form of the Raman response function $h_{\mathrm{R}}(t)$ :

$$
\begin{aligned}
h_{\mathrm{R}}(t)= & H(t)\left[(1-0.21) \tau_{1}\left(\tau_{1}^{-2}+\tau_{2}^{-2}\right) \exp \left(-t / \tau_{2}\right)\right. \\
& \left.\times \sin \left(t / \tau_{1}\right)+0.21\left(2 \tau_{b}-t\right) \tau_{b}^{-2} \exp \left(-t / \tau_{b}\right)\right],
\end{aligned}
$$

which is known to be well approximated by a damped harmonic oscillator response with $\tau_{1}=12.2 \mathrm{fs}, \tau_{2}=32 \mathrm{fs}$, and $\tau_{\mathrm{b}}=96 \mathrm{fs}$ [50]. The Heaviside $H(t)$ function in Eq. (3) reflects the property of causality of the Raman response function. Note that for the three different initial conditions considered in Figs. 4(a)-4(c), we have verified that the optical field evolves in the weakly nonlinear regime, i.e., $L_{\mathrm{D}}$ is typically smaller than $L_{\mathrm{NL}}$. This confirms the fact that the low-frequency MI sideband can act as a seed for the generation of SISs.

The RNLSE (2) has been integrated numerically to model the propagation of the wave between 0.75 and $21 \mathrm{~m}$ in the PCF for the three values of the input powers considered in Fig. 4. The initial condition refers to the previously fitted secant-hyperbolic spectrum (see Fig. 4) with uncorrelated random spectral phases. The assumption that the spectral components are $\delta$-correlated guarantees that the initial wave $A_{0}(t)=A(z=0.75 \mathrm{~m}, t)$ exhibits statistically stationary temporal fluctuations. This property of stationary statistics is a distinguished feature of SISs [17,19], and it has been also verified in the numerical simulations of the GNLSE discussed above through Fig. 2. The initial secant-hyperbolic spectrum is superimposed on a background of small noise, which mimics the quantum noise level (one photon per mode) [3]. Note that in the absence of such noise background, the SIS is known to undergo a slow adiabatic reshaping so as to adapt its shape to the local value of the background noise $[17,19]$.

The numerical simulations of the RNLSE (2) have been directly compared with the experimental spectrum for the three input powers considered in Fig. 4, and the corresponding results are reported in Figs. 5-7. We observe a remarkable qualitative agreement between the experimental evolution of the SIS's spectrum and the corresponding simulations of the RNLSE (2). Such a good agreement confirms the fact that the low-frequency branch of the spectrum is essentially ruled by the second-order dispersion and the Raman effect, which constitute the two necessary ingredients for the existence of SISs. We stress in particular that a transition occurs from the discrete SIS to the continuous SIS as the input power is increased, i.e., from Fig. 5 to Fig. 7. Unfortunately, in Fig. 7 the limited spectral window of our analyzer $(600-1750 \mathrm{~nm})$ allowed us to compare the experimental and numerical evolutions of the SIS over a limited spectral range. However, the agreement is remarkable, especially recalling the fact that the instantaneous Kerr effect has been neglected in the RNLSE. In particular, at small powers (Fig. 5), the evolution of the three spectral bands of the discrete SIS is almost in quantitative agreement with those recorded experimentally. Also, at high power (Fig. 7), we may note that the SIS's propagation in frequency space is almost rectilinear for $z>6 \mathrm{~m}$, as it should be for a genuine SIS.

\section{B. Kinetic equation}

The numerical simulations of the RNLSE (2) discussed above refer to a single realization of the initial random noise that models the partial coherence of the optical wave. Accordingly, the nonaveraged spectrum of the field is itself a stochastic function which, by nature, cannot describe a soliton behavior. To uncover the deterministic soliton behavior, one has to resort to a statistical description of the incoherent field, which is based on an average over the realizations $(\langle\rangle$.$) of$ the random wave. The corresponding kinetic equation $\mathrm{KE}$ describing the evolution of the averaged spectrum of the field was derived in Ref. [51] in the general case, and we refer the reader to this work for technical details.

The difficulty in the derivation of a KE relies on the fundamental problem of achieving a closure of the hierarchy of moments' equations. Indeed, because of the nonlinear character of the original GNLSE, the evolution of the second-order moment of the field [i.e., $B(z, t, \tau)=<A(z, t+\tau / 2) A^{*}(z, t-$ $\tau / 2)>$ ] depends on the corresponding fourth-order moment. In the same way, the equation for the fourth-order moment depends on the sixth-order moment, and so on. In this way, one obtains an infinite hierarchy of moment equations, in which the $n$ th-order moment depends on the $(n+2)$ th order moment of the field. This makes the equations impossible to solve unless some way can be found to truncate the hierarchy. A simple way to achieve a closure of the hierarchy is to assume that the optical field evolves in the weakly nonlinear regime of propagation, $L_{\mathrm{D}} \ll L_{\mathrm{NL}}$. In this regime the statistics of the optical field is approximately Gaussian, so that the property of factorizability of random Gaussian fields naturally leads to a closure of the hierarchy of moments' equations [13-16]. The weak-turbulence theory is essentially based on 

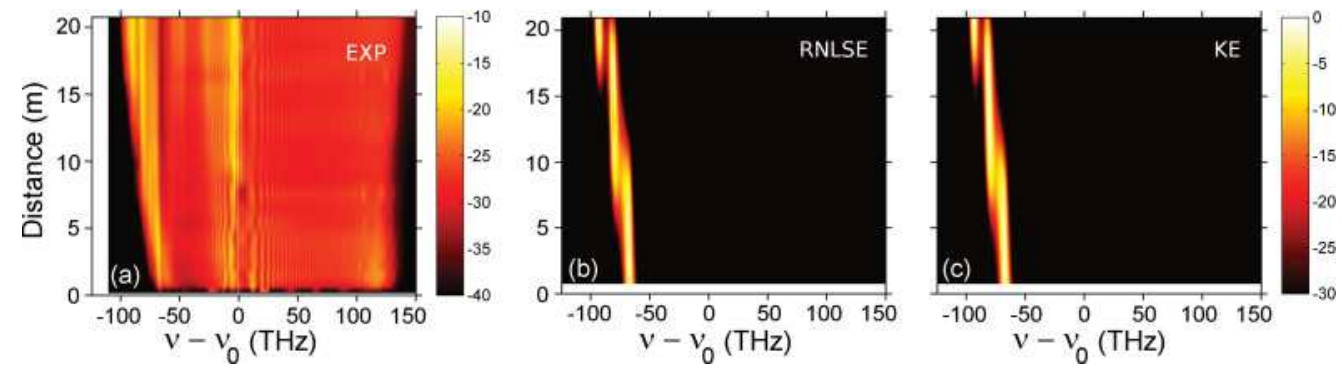

FIG. 5. (Color online) (a) Experimental results illustrating the spectral evolution (in $\mathrm{dB}$ scale) as a function of propagation distance in our 21-m-long PCF, for an input peak power about $1.32 \mathrm{~kW}$ [similar to Fig. 2(a)]. (b) Numerical simulations of the reduced RNLSE (2) starting with the secant-hyperbolic fit of Fig. 4(a). (c) Corresponding evolution of the averaged spectrum of the optical field, $n(z, \omega)$, obtained by integrating numerically the KE (4) with the same initial condition as in Fig. 5(b).

this approximation, which is usually called "random phase approximation" [13-16,51,52].

The weak turbulence KE can be derived from the RNLSE (2) and, in the general case, it exhibits a complicated form [51]. However, we verified in the numerical simulations that the optical field exhibits fluctuations that are statistically stationary, which means that the correlation function only depends on the time lag $\tau, B(z, \tau)$. Under the assumption of stationary statistics, the KE for the averaged spectrum, $n(z, \omega)=\int B(z, \tau) \exp [-i \omega \tau] d \tau$, takes the following simple form [17]:

$$
\frac{\partial n(z, \omega)}{\partial z}=\frac{\gamma}{\pi} n(z, \omega) \int_{-\infty}^{+\infty} g\left(\omega-\omega^{\prime}\right) n\left(z, \omega^{\prime}\right) d \omega^{\prime},
$$

where $g(\omega)=\operatorname{Im}\left[\tilde{\mathrm{h}}_{\mathrm{R}}(\omega)\right]$ is the imaginary part of the Fourier transform of the response function $h_{\mathrm{R}}(t)$, i.e., the Raman gain spectrum. Note that this kinetic equation does not account for dispersion effects [Eq. (4) does not depend on $\beta_{2}$ ], although the role of dispersion in its derivation is essential in order to verify the weakly nonlinear criterion, $L_{\mathrm{D}} / L_{\mathrm{NL}} \ll 1$. Then according to the KE (4), SISs are sustained in both the normal and the anomalous dispersion regime, a remarkable property that we have verified by direct numerical simulations of the RNLSE (2) in Ref. [19]. A more general form of the $\mathrm{KE}$ (4) has been the subject of a lot of studies in plasma physics, in particular to model weak Langmuir turbulence [20]. An analytical soliton solution to the KE (4) has been also derived in the context of stimulated Compton scattering [21].
This solution has been recently generalized in the context of optics in Ref. [51]. From a general point of view, a comparison between noninstantaneous and nonlocal nonlinear effects reveals that, in substance, the existence of SISs rely on the causality property of the Raman response function, a feature that was discussed in detail in Ref. [19].

The KE (4) has been recently shown to describe in detail all the properties of both discrete and continuous SISs [19]. In the following we show that it also describes the emergence of SIS observed in the SC experiment. Indeed, we performed numerical simulations of the KE (4) taking as initial conditions the secant-hyperbolic fit discussed above through Fig. 3. As for the RNLSE simulations, the secant-hyperbolic is superposed on a constant background whose amplitude is determined by the corresponding value of the averaged quantum noise. Note that we did not include any noise in the simulations of Eq. (4), since the KE describes the deterministic evolution of the averaged spectrum of the field. We report in Figs. 5(c), 6(c), and 7 (c) the corresponding evolutions of the spectra for the three different values of the power. We underline that a quantitative agreement is obtained between the simulations of the $\mathrm{KE}$ and the RNLSE, without using any adjustable parameter. In particular, the simulations confirm that a transition occurs from the discrete to the continuous SIS as the input power is increased. This transition may be intuitively interpreted as a consequence of the broadening of the initial MI spectral seed. For a spectral width of $3 \mathrm{THz}$ (Fig. 5), the optical field exhibits a discrete Raman shift simply because the low-frequency tail of the spectrum exhibits a much higher gain as compared to the
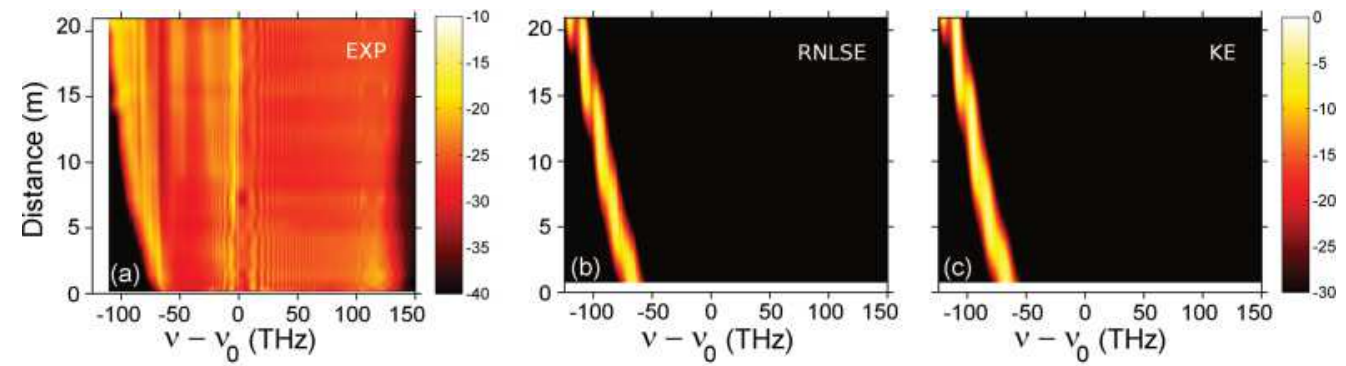

FIG. 6. (Color online) (a) Experimental results illustrating the spectral evolution (in dB scale) as a function of propagation distance in our 21-m-long PCF, for an input peak power about $2.22 \mathrm{~kW}$. (b) Numerical simulations of the reduced RNLSE (2) starting with the secant-hyperbolic fit of Fig. 4(b). (c) Corresponding evolution of the averaged spectrum of the optical field, $n(z, \omega)$, obtained by integrating numerically the KE (4) with the same initial condition as in Fig. 6(b). 

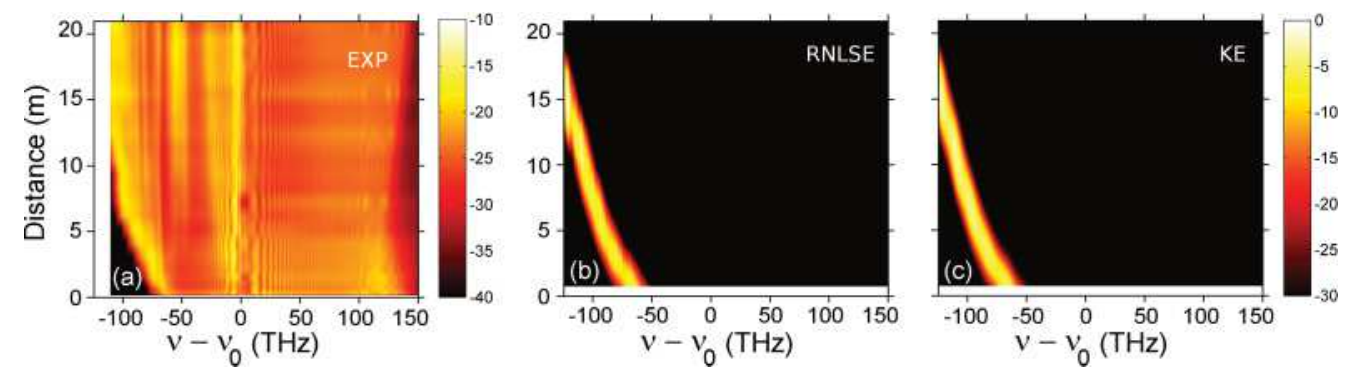

FIG. 7. (Color online) (a) Experimental results illustrating the spectral evolution (in dB scale) as a function of propagation distance in our 21-m-long PCF, for an input peak power about $4.52 \mathrm{~kW}$. (b) Numerical simulations of the reduced RNLSE (2) starting with the secant-hyperbolic fit of Fig. 4(c). (c) Corresponding evolution of the averaged spectrum of the optical field, $n(z, \omega)$, obtained by integrating numerically the KE (4) with the same initial condition as in Fig. 7(b).

mean gain of the whole front of the spectrum. Conversely, the Raman shift becomes continuous for the broad spectral width (9 THz) considered in Fig. 7.

It is important to underline that the continuous SIS behavior discussed in Fig. 7 only characterizes the first stage of the propagation of the optical field, i.e., $z<15 \mathrm{~m}$. Recalling that a power of $350 \mathrm{~W}$ is contained in the initial MI seed, this propagation length typically corresponds to 150 nonlinear lengths $L_{\mathrm{NL}}$. Although this propagation length is considerable, it corresponds only to the first stage of the SIS evolution. Indeed, the previous study reported in [19] revealed that continuous SISs can be extremely robust for very long propagation distances, while at some specific propagation length they may suddenly decay to a stable discrete SIS (see, e.g., Fig. 3 of [19]). This is indeed what happens in the example of Fig. 7, as revealed by the simulation of the KE reported in Fig. 8 for longer propagation distances. Actually, the parameter that determines the robustness of a continuous SIS is the amplitude of the noise background: As the background noise level decreases, the continuous SIS becomes unstable and relaxes toward its discrete counterpart [19]. In the experiment the amplitude of the noise background is determined by the quantum noise level, which is extremely small. Accordingly, the apparently stable behavior of the SIS reported in Figs. 7(b) and 7(c) refers in fact to a transient evolution, since the continuous SIS suddenly decays to a discrete SIS at $z \approx 20 \mathrm{~m}$

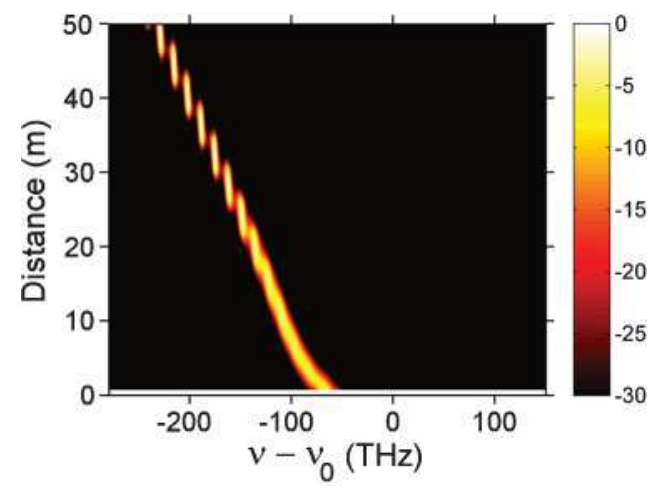

FIG. 8. (Color online) Numerical simulation of the KE (4) showing the long-term propagation of the simulation reported in Fig. 7(c). The continuous SIS is unstable and relaxes at $z \approx$ $20 \mathrm{~m}$ toward a discrete SIS during the propagation. (see Fig. 8). Actually, the issue of the stability properties of SISs is a difficult problem whose study goes beyond the present work and will be the subject of future investigations. Nevertheless, to conclude this section, we underline that the kinetic description of the experiment corroborates the previous study of the RNLSE (Sec. IV A) and thus confirms that the evolution of the incoherent structure identified in the low-frequency edge of the SC experiment can be interpreted as the emergence of a SIS.

\section{CONCLUSION}

In summary we have reported an experimental and numerical study of the emergence of SIS in the incoherent regime of SC generation in a two ZDWs PCF. An experimental cutback of the PCF has allowed us to perform detailed measurements of the evolution of the spectrum during the propagation of the field. The emergence of SIS from the SC spectrum has been analyzed in the framework of the reduced RNLSE and the weak-turbulence KE. It turns out that the KE captures the essential dynamical features that characterize the emergence of SIS through SC generation. In particular, the analysis reveals that in the first stage of propagation a transition from the discrete SIS toward the continuous SIS occurs as the power injected into the PCF is increased. On the other hand, although the continuous SIS is preserved for more than 100 nonlinear lengths $L_{\mathrm{NL}}$, it is shown to suddenly decay to a stable discrete SIS. In a loose sense, this suggests that continuous SIS may exhibit a kind of metastable behavior, a feature that will be the subject of future investigations.

\section{ACKNOWLEDGMENTS}

This work was supported by the Agence Nationale de la Recherche (ANR COSTUME and MANUREVA projects No. ANR-08-SYSC-004-03 and No. ANR-08-SYSC-019, respectively) and by the Conseil Régional de Bourgogne. A.K. and B.B. acknowledge financial support from the French Ministry of Higher Education and Research, the Nord-Pas de Calais Regional Council and FEDER through the "Contrat de Projets Etat Région (CPER) 2007-2013" and the "Campus Intelligence Ambiante" (CIA). 
[1] P. St. J. Russell, J. Lightwave Technol. 24, 4729 (2006).

[2] J. M. Dudley and J. R. Taylor, Nat. Photon. 3, 85 (2009).

[3] J. M. Dudley, G. Genty, and S. Coen, Rev. Mod. Phys. 78, 1135 (2006).

[4] G. Genty, S. Coen, and J. M. Dudley, J. Opt. Soc. Am. B 24, 1771 (2007).

[5] D. V. Skryabin and A. V. Gorbach, Rev. Mod. Phys. 82, 1287 (2010).

[6] J. M. Dudley and J. R. Taylor, Supercontinuum Generation in Optical Fibers (Cambridge University Press, Cambridge, 2010).

[7] W. J. Wadsworth, N. Joly, J. C. Knight, T. A. Birks, F. Biancalana, and P. St. J. Russell, Opt. Express 12, 299 (2004).

[8] B. Rumpf and A. C. Newell, Phys. Rev. Lett. 87, 054102 (2001).

[9] K. Hammani, B. Kibler, C. Finot, and A. Picozzi, Phys. Lett. A 374, 3585 (2010).

[10] B. Barviau, B. Kibler, S. Coen, and A. Picozzi, Opt. Lett. 33, 2833 (2008).

[11] B. Barviau, B. Kibler, A. Kudlinski, A. Mussot, G. Millot, and A. Picozzi, Opt. Express 17, 7392 (2009).

[12] B. Barviau, B. Kibler, and A. Picozzi, Phys. Rev. A 79, 063840 (2009).

[13] S. Dyachenko, A. C. Newell, A. Pushkarev, and V. E. Zakharov, Physica D 57, 96 (1992).

[14] V. Zakharov, V. L'vov, and G. Falkovich, Kolmogorov Spectra of Turbulence I (Springer, Berlin, 1992).

[15] A. C. Newell and B. Rumpf, Annu. Rev. Fluids Mech. 43, 59 (2001).

[16] For a simple introduction to wave turbulence theory in the context of nonlinear optics, see, e.g., A. Picozzi, Opt. Express 15, 9063 (2007).

[17] A. Picozzi, S. Pitois, and G. Millot, Phys. Rev. Lett. 101, 093901 (2008).

[18] A. V. Gorbach and D. V. Skryabin, Opt. Lett. 31, 3309 (2006).

[19] C. Michel, B. Kibler, and A. Picozzi, Phys. Rev. A 83, 023806 (2011).

[20] S. L. Musher, A. M. Rubenchik, and V. E. Zakharov, Phys. Rep. 252, 177 (1995).

[21] C. Montes, Phys. Rev. A 20, 1081 (1979).

[22] A. Picozzi, Opt. Lett. 29, 1653 (2004).

[23] B. Barviau, S. Randoux, and P. Suret, Opt. Lett. 31, 1696 (2006).

[24] D. V. Dylov and J. W. Fleischer, Phys. Rev. Lett. 100, 103903 (2008).

[25] A. Picozzi and M. Haelterman, Phys. Rev. Lett. 92, 103901 (2004).

[26] A. Picozzi and M. Haelterman, Phys. Rev. Lett. 88, 083901 (2002).

[27] S. Pitois, S. Lagrange, H. R. Jauslin, and A. Picozzi, Phys. Rev. Lett. 97, 033902 (2006).
[28] L. Levi, T. Schwartz, O. Manela, M. Segev, and H. Buljan, Opt. Express 16, 7818 (2008)

[29] A. Picozzi and S. Rica, Europhys. Lett. 84, 34004 (2008).

[30] Y. Silberberg, Y. Lahini, Y. Bromberg, E. Small, and R. Morandotti, Phys. Rev. Lett. 102, 233904 (2009).

[31] C. Barsi, W. Wan, and J. W. Fleischer, Nat. Photon. 3, 211 (2009).

[32] C. Conti, M. Leonetti, A. Fratalocchi, L. Angelani, and G. Ruocco, Phys. Rev. Lett. 101, 143901 (2008).

[33] S. A. Babin, D. V. Churkin, A. E. Ismagulov, S. I. Kablukov, and E. V. Podivilov, J. Opt. Soc. Am. B 24, 1729 (2007).

[34] S. A. Babin, V. Karalekas, E. V. Podivilov, V. K. Mezentsev, P. Harper, J. D. Ania-Castañón, and S. K. Turitsyn, Phys. Rev. A 77, 033803 (2008).

[35] D. V. Churkin, S. A. Babin, A. E. El-Taher, P. Harper, S. I. Kablukov, V. Karalekas, J. D. Ania-Castañón, E. V. Podivilov, and S. K. Turitsyn, Phys. Rev. A 82, 033828 (2010).

[36] S. K. Turitsyn, S. A. Babin, A. E. El-Taher, P. Harper, D. V. Churkin, S. I. Kablukov, J. D. Ania-Castañon, V. Karalekas, and E. V. Podivilov, Nat. Photon. 4, 231 (2010).

[37] R. Weill, B. Fischer, and O. Gat, Phys. Rev. Lett. 104, 173901 (2010).

[38] R. Weill, B. Levit, A. Bekker, O. Gat, and B. Fischer, Opt. Express 18, 16520 (2010).

[39] J. Klaers, F. Vewinger, and M. Weitz, Nat. Phys. 6, 512 (2010).

[40] J. Klaers, J. Schmitt, F. Vewinger, and M. Weitz, Nature 468, 545 (2010).

[41] Y. Bromberg, Y. Lahini, E. Small, and Y. Silberberg, Nat. Photon. 4, 721 (2010).

[42] A. Picozzi, Opt. Express 16, 17171 (2008).

[43] P. Suret, S. Randoux, H. R. Jauslin, and A. Picozzi, Phys. Rev. Lett. 104, 054101 (2010).

[44] C. Michel, P. Suret, S. Randoux, H. R. Jauslin, and A. Picozzi, Opt. Lett. 35, 2367 (2010).

[45] D. Soh, J. P. Koplow, S. W. Moore, K. L. Schroder, and W. L. Hsu, Opt. Express 18, 22393 (2010).

[46] S. Lagrange, H. R. Jauslin, and A. Picozzi, Europhys. Lett. 79, 64001 (2007).

[47] U. Bortolozzo, J. Laurie, S. Nazarenko, and S. Residori, J. Opt. Soc. Am. B 26, 2280 (2009).

[48] G. P. Agrawal, Nonlinear Fiber Optics, 4th ed. (Academic, New York, 2006).

[49] P. M. Moselund, M. H. Frosz, C. L. Thomsen, and O. Bang, Opt. Express 16, 11954 (2008).

[50] Q. Lin and G. P. Agrawal, Opt. Lett. 31, 3086 (2006).

[51] J. Garnier and A. Picozzi, Phys. Rev. A 81, 033831 (2010).

[52] V. E. Zakharov, F. Dias, and A. Pushkarev, Phys. Rep. 398, 1 (2004). 